\title{
New Integrable and Linearizable Nonlinear Difference Equations
}

R. Sahadevan, G. Nagavigneshwari

To cite this article: R. Sahadevan, G. Nagavigneshwari (2013) New Integrable and Linearizable Nonlinear Difference Equations, Journal of Nonlinear Mathematical Physics 20:2, 179-190, DOI: https://doi.org/10.1080/14029251.2013.805563

To link to this article: https://doi.org/10.1080/14029251.2013.805563

Published online: 04 January 2021 


\title{
New Integrable and Linearizable Nonlinear Difference Equations
}

\author{
R. Sahadevan and G. Nagavigneshwari \\ Ramanujan Institute for Advanced Study in Mathematics, \\ University of Madras, Chennai-600 005, Tamil Nadu, India \\ ramajayamsaha@yahoo.co.in,grgnaga@gmail.com \\ Received 11 October 2012 \\ Accepted 7 January 2013
}

\begin{abstract}
A systematic investigation to derive nonlinear lattice equations governed by partial difference equations $(P \Delta \Delta E)$ admitting specific Lax representation is presented. Further it is shown that for a specific value of the parameter the derived nonlinear $P \Delta \Delta E$ 's can be transformed into a linear $P \Delta \Delta E$ 's under a global transformation. Also it is demonstrated how to derive higher order ordinary difference equations $(O \Delta E)$ or mappings in general and linearizable ones in particular from the obtained nonlinear $P \Delta \Delta E$ 's through periodic reduction. The question of measure preserving property of the obtained $O \Delta E$ 's and the construction of more than one integrals (or invariants) of them is examined wherever possible.
\end{abstract}

PACS: 02.30.Ik

Mathematical Subject Classification: 39A14

\section{Introduction}

The study of discrete systems governed by nonlinear $P \Delta \Delta E$ s and $O \Delta E$ s has attracted researchers in nonlinear phenomena in recent years. One of the reasons for the interest to study discrete systems is that they are more fundamental than the continuous ones. Also it is of interest to understand whether or not the discrete systems derived from continuous nonlinear systems governed by ordinary or partial differential equation especially integrable ones preserve their integrability characteristics $[1,2,5,11,20-26]$. In the last few decades, considerable progress has been accomplished and several integrable nonlinear ordinary and partial differential equations were discretized leading to differential-difference, ordinary difference equations or mappings preserving integrability characteristics of their counterpart [7, 12, 14, 27-29, 33, 34]. Several analytical methods have also been devised to derive both mathematical and physical aspects from integrability to chaos of discrete nonlinear systems. To the best of our knowledge only a few nonlinear partial difference equations or lattice equations with two independent variables have been derived whose continuum limit can be related with known integrable partial differential equations with two independent variables including soliton possessing systems. It is appropriate to mention that the study of integrable nonlinear $P \Delta \Delta E$ 's enables one to derive integrable higher dimensional nonlinear $O \Delta E$ 's or integrable mappings, for example through periodic reductions $[3,8,16,18,19,21]$ and hence the search for integrable lattice equations involving two or more independent variables is interesting. 
It is known that the concept of integrability of nonlinear difference equations is not well defined like for nonlinear differential equations however there exists some working definitions in the literature. A nonlinear $P \Delta \Delta E$ with two independent variables is said to be integrable

(i) if it arises from the compatibility condition of a system of linear partial difference equations $[1,2,18]$ and the underlying method is referred to as Lax pair method;

(ii) if it possesses multi-soliton solutions $[11,13,19]$;

(iii) if it passes the ultra-local singularity confinement criterion $[10,11]$ and has zero algebraic entropy $[6,15]$;

(iv) if it has the Consistency Around the Cube (CAC) property [4];

(v) if it can be transformed into a linear partial difference equation through a global transformation;

We would like to mention that in definition (v), the transformation of a nonlinear differential equation means that the solutions can be expressed in terms of known functions. Hence in the discrete case also one would expect the discrete analogues of the known functions to play a crucial role. In this article a scalar nonlinear $P \Delta \Delta E$ with two independent variables having the form

$$
v_{m+1}^{l+1}=F\left(v_{m}^{l}, v_{m}^{l+1}, v_{m+1}^{l}\right), v_{m}^{l}=v(l, m)
$$

is considered and derived equations admitting specific Lax representation. The identified nonlinear $P \Delta \Delta E$ 's can be classified into two distinct forms namely

$$
\text { (i) } \quad v_{m+1}^{l+1}=\frac{h_{11}\left(v_{m}^{l}\right)^{3}+h_{12}\left(v_{m}^{l}\right)^{2}+h_{13} v_{m}^{l}+h_{14}}{h_{15}\left(v_{m}^{l}\right)^{3}+h_{16}\left(v_{m}^{l}\right)^{2}+h_{17} v_{m}^{l}+h_{18}}
$$

and

$$
v_{m+1}^{l+1}=\frac{F_{11}\left(v_{m}^{l+1}, v_{m+1}^{l}\right)+v_{m}^{l} F_{12}\left(v_{m}^{l+1}, v_{m+1}^{l}\right)}{F_{12}\left(v_{m}^{l+1}, v_{m+1}^{l}\right)-v_{m}^{l} F_{11}\left(v_{m}^{l+1}, v_{m+1}^{l}\right)}
$$

where $h_{1 i}$ 's are polynomials in $\left(v_{m}^{l+1}, v_{m+1}^{l}\right)$ and $F_{11}$ and $F_{12}$ are polynomials of degree $(2|r|-1)$ and $2|r|$ respectively, $r \in \mathbb{Z} \backslash\{0\}$. It is shown that the latter nonlinear $P \Delta \Delta E$ with specific forms of $F_{1 i} i=1,2$ can be transformed into linear $P \Delta \Delta E$ through a global transformation.

The plan of the article is as follows. Given a set of Lax pairs with rational entries having the form, $\frac{P}{Q}$ where both $P$ and $Q$ are polynomials in $v_{m}^{l}, v_{m}^{l+1}$ and $v_{m+1}^{l}$, how to derive the associated nonlinear $P \Delta \Delta E$ 's with two independent variables is presented in section 2 which results to the above equations (1.1) and (1.2). It is shown that equation (1.2) is linearizable under a global transformation ensuring its integrability. In section 3 it is demonstrated how to derive higher order $O \Delta E$ 's in general and linearizable ones in particular from the obtained nonlinear $P \Delta \Delta E$ 's through periodic reductions. The question of measure preserving property of the obtained $O \Delta E$ 's and construction of more than one integrals of them is examined wherever possible. In section 4 a brief summary of the obtained results and concluding remarks are presented.

\section{Integrable and Linearizable Nonlinear Partial Difference Equations}

Consider a system of linear difference equations with two independent variables $l$ and $m$ given by

$$
\left(\begin{array}{l}
v_{1 m}^{l+1} \\
v_{2 m}^{l+1}
\end{array}\right)=L(l, m, k)\left(\begin{array}{l}
v_{1 m}^{l} \\
v_{2 m}^{l}
\end{array}\right)
$$




$$
\left(\begin{array}{l}
v_{1 m+1}^{l} \\
v_{2 m+1}^{l}
\end{array}\right)=M(l, m, k)\left(\begin{array}{l}
v_{1 m}^{l} \\
v_{2 m}^{l}
\end{array}\right)
$$

where $L(l, m, k)$ and $M(l, m, k)$ denoted by $L_{m}^{l}$ and $M_{m}^{l}$ are $(2 \times 2)$ matrices. Here $v_{1 m}^{l}$ and $v_{2 m}^{l}$ are wave functions defined at the sites of a two-dimensional lattice as functions of the spectral parameter $k$. Then the compatibility condition of (2.1) and (2.2) gives

$$
M_{m}^{l+1} L_{m}^{l}=L_{m+1}^{l} M_{m}^{l}
$$

which is usually referred to as Lax equation. Let us assume that the matrices $L_{m}^{l}$ and $M_{m}^{l}$ depend only on the potential $\left(v_{m}^{l}, v_{m}^{l+1}\right)$ and $\left(v_{m}^{l}, v_{m+1}^{l}\right)$ respectively. Then the Lax equation (2.3) is equivalent to a condition of the type

$$
v_{m+1}^{l+1}=F\left(v_{m}^{l}, v_{m}^{l+1}, v_{m+1}^{l}\right) .
$$

Recently we considered Lax matrices with rational entries, having the form $\frac{P}{Q}$ where both $P$ and $Q$ are linear in $v_{m}^{l}, v_{m}^{l+1}$ and $v_{m+1}^{l}$ and reported several new nonlinear $P \Delta \Delta E$ 's satisfying (2.3) and hence they are integrable in the sense of Lax [30]. In this article we wish to consider Lax matrices again with rational entries in which $P$ and $Q$ are algebraic polynomials of degree greater than one and show that there exists a class of nonlinear $P \Delta \Delta E$ 's satisfying the compatibility condition (2.3).

We now consider specific Lax matrices $L_{m}^{l}$ and $M_{m}^{l}$ having the form

$$
\begin{aligned}
& L_{m}^{l}(k)=\left(\begin{array}{cc}
0 & \frac{f_{11}\left(v_{m}^{l}, v_{m}^{l+1}\right)}{k f_{12}\left(v_{m}^{l}, v_{m}^{l+1}\right)} \\
\frac{k f_{13}\left(v_{m}^{l}, v_{m}^{l+1}\right)}{f_{14}\left(v_{m}^{l}, v_{m}^{l+1}\right)} & 0
\end{array}\right), \\
& M_{m}^{l}(k)=\left(\begin{array}{cc}
0 & \frac{g_{11}\left(v_{m}^{l}, v_{m+1}^{l}\right)}{k g_{12}\left(v_{m}^{l}, v_{m+1}^{l}\right)} \\
\frac{k g_{13}\left(v_{m}^{l}, v_{m+1}^{l}\right)}{g_{14}\left(v_{m}^{l}, v_{m+1}^{l}\right)} & 0
\end{array}\right),
\end{aligned}
$$

where $k$ is the spectral parameter and $f_{1 i}$ 's and $g_{1 i}$ 's, $i=1,2,3$ and 4 are arbitrary unknown functions. It is easy to verify that the components $(1,1)$ and $(2,2)$ of the compatibility condition $(2.3)$ vanish while the components of $(1,2)$ and $(2,1)$ result the following respectively:

$$
\begin{aligned}
& \frac{f_{11}\left(v_{m+1}^{l}, v_{m+1}^{l+1}\right) f_{14}\left(v_{m}^{l}, v_{m}^{l+1}\right)}{f_{12}\left(v_{m+1}^{l}, v_{m+1}^{l+1}\right) f_{13}\left(v_{m}^{l}, v_{m}^{l+1}\right)}=\frac{g_{11}\left(v_{m}^{l+1}, v_{m+1}^{l+1}\right) g_{14}\left(v_{m}^{l}, v_{m+1}^{l}\right)}{g_{12}\left(v_{m}^{l+1}, v_{m+1}^{l+1}\right) g_{13}\left(v_{m}^{l}, v_{m+1}^{l}\right)}, \\
& \frac{f_{13}\left(v_{m+1}^{l}, v_{m+1}^{l+1}\right) f_{12}\left(v_{m}^{l}, v_{m}^{l+1}\right)}{f_{14}\left(v_{m+1}^{l}, v_{m+1}^{l+1}\right) f_{11}\left(v_{m}^{l}, v_{m}^{l+1}\right)}=\frac{g_{13}\left(v_{m}^{l+1}, v_{m+1}^{l+1}\right) g_{12}\left(v_{m}^{l}, v_{m+1}^{l}\right)}{g_{14}\left(v_{m}^{l+1}, v_{m+1}^{l+1}\right) g_{11}\left(v_{m}^{l}, v_{m+1}^{l}\right)} .
\end{aligned}
$$

From equations (2.7) and (2.8) it is clear that they reduce into a single equation

$$
\frac{f_{11}\left(v_{m+1}^{l}, v_{m+1}^{l+1}\right) f_{12}\left(v_{m}^{l}, v_{m}^{l+1}\right)}{f_{12}\left(v_{m+1}^{l}, v_{m+1}^{l+1}\right) f_{11}\left(v_{m}^{l}, v_{m}^{l+1}\right)}=\frac{g_{11}\left(v_{m}^{l+1}, v_{m+1}^{l+1}\right) g_{12}\left(v_{m}^{l}, v_{m+1}^{l}\right)}{g_{12}\left(v_{m}^{l+1}, v_{m+1}^{l+1}\right) g_{11}\left(v_{m}^{l}, v_{m+1}^{l}\right)},
$$

provided

$$
\begin{aligned}
& f_{13}\left(v_{m}^{l}, v_{m}^{l+1}\right)=f_{11}\left(v_{m}^{l}, v_{m}^{l+1}\right), f_{14}\left(v_{m}^{l}, v_{m}^{l+1}\right)=f_{12}\left(v_{m}^{l}, v_{m}^{l+1}\right), \\
& g_{13}\left(v_{m}^{l}, v_{m+1}^{l}\right)=g_{11}\left(v_{m}^{l}, v_{m+1}^{l}\right), g_{14}\left(v_{m}^{l}, v_{m+1}^{l}\right)=g_{12}\left(v_{m}^{l}, v_{m+1}^{l}\right) .
\end{aligned}
$$


Equation (2.9) is a functional equation and therefore cannot be solved for $v_{m+1}^{l+1}$ explicitly. To solve equation (2.9) for $v_{m+1}^{l+1}$ we first consider $f_{1 i}$ 's and $g_{1 i}$ 's, $i=1,2$, are linear which leads to not so interesting cases. Next we consider both $f_{1 i}$ 's and $g_{1 i}$ 's are quadratic in $\left(v_{m}^{l}, v_{m}^{l+1}, v_{m+1}^{l}\right)$. A detailed calculation show that equation (2.9) can be solved for $v_{m+1}^{l+1}$, for the following forms:

$f_{11}\left(v_{m}^{l}, v_{m}^{l+1}\right)=\left(\alpha+v_{m}^{l}\right)^{2}, \quad f_{12}\left(v_{m}^{l}, v_{m}^{l+1}\right)=\left(\alpha v_{m}^{l}+1\right)^{2}$ $g_{11}\left(v_{m}^{l}, v_{m+1}^{l}\right)=\left(-\alpha v_{m}^{l}+1\right)\left(\alpha-v_{m+1}^{l}\right), g_{12}\left(v_{m}^{l}, v_{m+1}^{l}\right)=\left(\alpha-v_{m}^{l}\right)\left(\alpha+v_{m+1}^{l}\right)$,

where $\alpha$ is an arbitrary parameter and so we obtain a nonlinear $P \Delta \Delta E$ which can be written as a ratio of polynomials of degree three in $v_{m}^{l}$

$$
v_{m+1}^{l+1}=\frac{h_{11}\left(v_{m}^{l}\right)^{3}+h_{12}\left(v_{m}^{l}\right)^{2}+h_{13} v_{m}^{l}+h_{14}}{h_{15}\left(v_{m}^{l}\right)^{3}+h_{16}\left(v_{m}^{l}\right)^{2}+h_{17} v_{m}^{l}+h_{18}}
$$

where $h_{1 i}$ 's, $i=1, \ldots 8$ are also polynomials in $\left(v_{m}^{l+1}, v_{m+1}^{l}\right)$,

$$
\begin{aligned}
h_{11}= & \alpha\left[1-\alpha^{6}+\alpha\left(\alpha^{4}-1\right) v_{m}^{l+1}+2 \alpha v_{m+1}^{l}-2 \alpha^{2}\right. \\
& \left.v_{m}^{l+1} v_{m+1}^{l}+\alpha^{2}\left(\alpha^{2}+1\right)\left(v_{m+1}^{l}\right)^{2}-2 \alpha^{3} v_{m}^{l+1}\left(v_{m+1}^{l}\right)^{2}\right] \\
h_{12}= & \alpha^{2}\left[\left(1-\alpha^{4}\right)+\alpha\left(\alpha^{2}-1\right) v_{m}^{l+1}+2 \alpha v_{m+1}^{l}-2 \alpha^{2} v_{m}^{l+1} v_{m+1}^{l}\right. \\
& \left.+2 \alpha^{2}\left(v_{m+1}^{l}\right)^{2}-\alpha\left(\alpha^{2}+1\right) v_{m}^{l+1}\left(v_{m+1}^{l}\right)^{2}\right] \\
h_{13}= & \alpha^{2}\left[\alpha\left(\alpha^{2}-1\right)-2 \alpha^{2} v_{m+1}^{l}+2 \alpha^{3} v_{m}^{l+1} v_{m+1}^{l}\right. \\
& \left.-\alpha\left(\alpha^{2}+1\right)\left(v_{m+1}^{l}\right)^{2}+\left(\alpha^{4}+1\right) v_{m}^{l+1}\left(v_{m+1}^{l}\right)^{2}\right], \\
h_{14}= & \alpha\left[\alpha^{2}\left(\alpha^{2}-1\right) v_{m}^{l+1}-2 \alpha^{4} v_{m+1}^{l}+2 \alpha^{5} v_{m}^{l+1} v_{m+1}^{l}\right. \\
& \left.-\alpha\left(\alpha^{4}+1\right)\left(v_{m+1}^{l}\right)^{2}+\left(\alpha^{6}+1\right) v_{m}^{l+1}\left(v_{m+1}^{l}\right)^{2}\right] \\
h_{15}= & \left(\alpha^{6}+1\right)-\alpha\left(\alpha^{4}+1\right) v_{m}^{l+1}+2 \alpha v_{m+1}^{l}-2 \alpha^{2} v_{m}^{l+1} v_{m+1}^{l} \\
& -\alpha^{2}\left(\alpha^{2}-1\right)\left(v_{m+1}^{l}\right)^{2} \\
h_{16}= & \alpha\left[\left(\alpha^{4}+1\right)-\alpha\left(\alpha^{2}+1\right) v_{m}^{l+1}+2 \alpha v_{m+1}^{l}-2 \alpha^{2} v_{m}^{l+1} v_{m+1}^{l}\right. \\
& \left.-\alpha\left(\alpha^{2}-1\right) v_{m}^{l+1}\left(v_{m+1}^{l}\right)^{2}\right] \\
h_{17}= & \alpha\left[-\alpha\left(\alpha^{2}+1\right)+2 \alpha^{2}\left(v_{m}^{l+1}-v_{m+1}^{l}\right)+2 \alpha^{3} v_{m}^{l+1} v_{m+1}^{l}\right. \\
& \left.-\alpha\left(\alpha^{2}-1\right)\left(v_{m+1}^{l}\right)^{2}+\left(\alpha^{4}-1\right) v_{m}^{l+1}\left(v_{m+1}^{l}\right)^{2}\right], \\
h_{18}= & -2 \alpha^{3}+\alpha^{2}\left(\alpha^{2}+1\right) v_{m}^{l+1}-2 \alpha^{4} v_{m+1}^{l}+2 \alpha^{5} v_{m}^{l+1} v_{m+1}^{l} \\
& -\alpha\left(\alpha^{4}-1\right)\left(v_{m+1}^{l}\right)^{2}+\left(\alpha^{6}-1\right) v_{m}^{l+1}\left(v_{m+1}^{l}\right)^{2} .
\end{aligned}
$$

Thus the equation (2.10) is integrable in the sense of Lax. It is not clear, at the moment, whether this equation possesses other characteristics of integrability such as CAC (Consistency Around the Cube) property, ultra-singularity confinement criteria, conservation laws, etc. However we wish to report that when $\alpha^{2}=-1$, equation (2.10) becomes

$$
v_{m+1}^{l+1}=\frac{2\left(i-v_{m+1}^{l}\right)\left(\left(v_{m}^{l}\right)^{2}+1\right)\left[v_{m}^{l+1}-v_{m+1}^{l}+v_{m}^{l}\left(1+v_{m}^{l+1} v_{m+1}^{l}\right)\right]}{2\left(i-v_{m+1}^{l}\right)\left(\left(v_{m}^{l}\right)^{2}+1\right)\left[\left(1+v_{m}^{l+1} v_{m+1}^{l}\right)-v_{m}^{l}\left(v_{m}^{l+1}-v_{m+1}^{l}\right)\right]}
$$

which can be transformed into a linear $P \Delta \Delta E$

$$
\theta_{m+1}^{l+1}-\theta_{m}^{l+1}+\theta_{m+1}^{l}-\theta_{m}^{l}=p \pi, \theta_{m}^{l}=\tan ^{-1}\left(v_{m}^{l}\right), p \in \mathbb{Z}
$$

and thus equation (2.12) is both integrable and linearizable.

Next we consider $f_{1 i}$ 's and $g_{1 i}$ 's are polynomials of degree three in their respective arguments. A detailed calculation show that equation (2.9) is solvable for $v_{m+1}^{l+1}$ when the degree of $g_{1 i}$ is lesser by 
2 than that of $f_{1 i}$. In other words, for the following forms

$$
\begin{array}{ll}
f_{11}\left(v_{m}^{l}, v_{m}^{l+1}\right)=\left(\alpha+v_{m}^{l}\right)^{2}\left(\alpha+v_{m}^{l+1}\right), & f_{12}\left(v_{m}^{l}, v_{m}^{l+1}\right)=\left(\alpha v_{m}^{l}+1\right)^{2}\left(\alpha v_{m}^{l+1}+1\right), \\
g_{11}\left(v_{m}^{l}, v_{m+1}^{l}\right)=\left(-\alpha v_{m}^{l}+1\right), & g_{12}\left(v_{m}^{l}, v_{m+1}^{l}\right)=\left(\alpha-v_{m}^{l}\right)
\end{array}
$$

where $\alpha$ is an arbitrary parameter, equation (2.9) yields a nonlinear $P \Delta \Delta E$ which can be written as ratio of polynomials of degree three in $v_{m}^{l}$

$$
v_{m+1}^{l+1}=\frac{\tilde{h}_{11}\left(v_{m}^{l}\right)^{3}+\tilde{h}_{12}\left(v_{m}^{l}\right)^{2}+\tilde{h}_{13} v_{m}^{l}+\tilde{h}_{14}}{\tilde{h}_{15}\left(v_{m}^{l}\right)^{3}+\tilde{h}_{16}\left(v_{m}^{l}\right)^{2}+\tilde{h}_{17} v_{m}^{l}+\tilde{h}_{18}}
$$

where $\tilde{h}_{1 i}$ 's, $i=1, \ldots 8$ are also polynomials in $\left(v_{m}^{l+1}, v_{m+1}^{l}\right)$. Since each $\tilde{h}_{1 i}$ 's involve a lengthy expression we refrain from presenting its explicit form. Thus the equation (2.14) is integrable in the sense of Lax. Here again for $\alpha^{2}=-1$, equation (2.14), after eliminating common factors appeared in both numerator and the denominator, becomes a QRT type equation,

$$
v_{m+1}^{l+1}=\frac{F_{11}\left(v_{m}^{l+1}, v_{m+1}^{l}\right)+v_{m}^{l} F_{12}\left(v_{m}^{l+1}, v_{m+1}^{l}\right)}{F_{12}\left(v_{m}^{l+1}, v_{m+1}^{l}\right)-v_{m}^{l} F_{11}\left(v_{m}^{l+1}, v_{m+1}^{l}\right)},
$$

where

$F_{11}=2\left(v_{m}^{l+1}-v_{m+1}^{l}\right)\left(1+v_{m}^{l+1} v_{m+1}^{l}\right)$,
$F_{12}=\left(1+v_{m}^{l+1}-v_{m+1}^{l}+v_{m}^{l+1} v_{m+1}^{l}\right)\left(1-v_{m}^{l+1}+v_{m+1}^{l}+v_{m}^{l+1} v_{m+1}^{l}\right)$.

which can also be transformed into a linear $P \Delta \Delta E$

$$
\theta_{m+1}^{l+1}-2\left(\theta_{m}^{l+1}-\theta_{m+1}^{l}\right)-\theta_{m}^{l}=p \pi, \theta_{m}^{l}=\tan ^{-1}\left(v_{m}^{l}\right), p \in \mathbb{Z}
$$

and hence integrable and linearizable as well.

Next we consider $f_{1 i}$ 's and $g_{1 i}$ 's are polynomials of degree four in their respective arguments and find that the solution of (2.9), that is for $v_{m+1}^{l+1}$ involve irrational functions and hence not pursued further. The situation remains the same when $f_{1 i}$ 's and $g_{1 i}$ 's are polynomials of degree $>4$. However for the following forms of $f_{1 i}$ 's and $g_{1 i}$ 's

$$
\left.\begin{array}{l}
f_{11}\left(v_{m}^{l}, v_{m}^{l+1}\right)=\left(\alpha+v_{m}^{l}\right)^{2}\left(\alpha+v_{m}^{l+1}\right)^{r-1}, \\
f_{12}\left(v_{m}^{l}, v_{m}^{l+1}\right)=\left(\alpha v_{m}^{l}+1\right)^{2}\left(\alpha v_{m}^{l+1}+1\right)^{r-1}, \\
g_{11}\left(v_{m}^{l}, v_{m+1}^{l}\right)=\left(-\alpha v_{m}^{l}+1\right)\left(\alpha+v_{m+1}^{l}\right)^{r-2}, \\
g_{12}\left(v_{m}^{l}, v_{m+1}^{l}\right)=\left(\alpha-v_{m}^{l}\right)\left(\alpha-v_{m+1}^{l}\right)^{r-2}
\end{array}\right\}
$$

with $\alpha^{2}=-1$, equation (2.9) leads to QRT type nonlinear $P \Delta \Delta E$ of the form

$$
v_{m+1}^{l+1}=\frac{F_{11}\left(v_{m}^{l+1}, v_{m+1}^{l}\right)+v_{m}^{l} F_{12}\left(v_{m}^{l+1}, v_{m+1}^{l}\right)}{F_{12}\left(v_{m}^{l+1}, v_{m+1}^{l}\right)-v_{m}^{l} F_{11}\left(v_{m}^{l+1}, v_{m+1}^{l}\right)},
$$

where $F_{11}$ and $F_{12}$ are specific polynomials of degree $(2|r|-1)$ and $2|r|$ respectively and their explicit expression for different values of $r$ are given in Table 1 and Table 2.

Here again equation (2.18) can be transformed into a linear $P \Delta \Delta E$

$$
\theta_{m+1}^{l+1}-r\left(\theta_{m}^{l+1}-\theta_{m+1}^{l}\right)-\theta_{m}^{l}=p \pi, \theta_{m}^{l}=\tan ^{-1}\left(v_{m}^{l}\right), p \in \mathbb{Z}, r \in \mathbb{Z} \backslash\{0\} .
$$

Hence equation (2.18) is integrable in the sense of Lax as well as being linearizable under a global transformation. 


\begin{tabular}{|c|c|}
\hline$r$ & Explicit expressions of $F_{11}$ and $F_{12}$ in equation (2.18) \\
\hline$r=1$ & $\begin{array}{l}F_{11}=\left(v_{m}^{l+1}-v_{m+1}^{l}\right) \\
F_{12}=\left(1+v_{m}^{l+1} v_{m+1}^{l}\right)\end{array}$ \\
\hline$r=2$ & $\begin{array}{l}F_{11}=2\left(v_{m}^{l+1}-v_{m+1}^{l}\right)\left(1+v_{m}^{l+1} v_{m+1}^{l}\right) \\
F_{12}=\left(1+v_{m}^{l+1}-v_{m+1}^{l}+v_{m}^{l+1} v_{m+1}^{l}\right)\left(1-v_{m}^{l+1}+v_{m+1}^{l}+v_{m}^{l+1} v_{m+1}^{l}\right)\end{array}$ \\
\hline$r=3$ & $\begin{array}{l}F_{11}=\left(v_{m}^{l+1}-v_{m+1}^{l}\right)\left[3-\left(v_{m}^{l+1}\right)^{2}-\left(v_{m+1}^{l}\right)^{2}+8 v_{m}^{l+1} v_{m+1}^{l}+3\left(v_{m}^{l+1}\right)^{2}\left(v_{m+1}^{l}\right)^{2}\right] \\
F_{12}=\left(1+v_{m}^{l+1} v_{m+1}^{l}\right)\left[1-3\left(v_{m}^{l+1}\right)^{2}-3\left(v_{m+1}^{l}\right)^{2}+8 v_{m}^{l+1} v_{m+1}^{l}+\left(v_{m}^{l+1}\right)^{2}\left(v_{m+1}^{l}\right)^{2}\right]\end{array}$ \\
\hline$r=4$ & $\begin{aligned} F_{11}= & 2\left(v_{m}^{l+1}-v_{m+1}^{l}\right)\left(1+v_{m}^{l+1} v_{m+1}^{l}\right)\left(1+v_{m}^{l+1}-v_{m+1}^{l}+v_{m}^{l+1} v_{m+1}^{l}\right) \\
& \left(1-v_{m}^{l+1}+v_{m+1}^{l}+v_{m}^{l+1} v_{m+1}^{l}\right) \\
F_{12}= & {\left[1+2\left(v_{m}^{l+1}-v_{m+1}^{l}\right)-\left(v_{m}^{l+1}\right)^{2}-\left(v_{m+1}^{l}\right)^{2}+4 v_{m}^{l+1} v_{m+1}^{l}\right.} \\
& \left.+2 v_{m}^{l+1} v_{m+1}^{l}\left(v_{m}^{l+1}-v_{m+1}^{l}\right)+\left(v_{m}^{l+1}\right)^{2}\left(v_{m+1}^{l}\right)^{2}\right]\left[1-2\left(v_{m}^{l+1}-v_{m+1}^{l}\right)\right. \\
& -\left(v_{m}^{l+1}\right)^{2}-\left(v_{m+1}^{l}\right)^{2}+4 v_{m}^{l+1} v_{m+1}^{l}-2 v_{m}^{l+1} v_{m+1}^{l}\left(v_{m}^{l+1}-v_{m+1}^{l}\right) \\
& \left.+\left(v_{m}^{l+1}\right)^{2}\left(v_{m+1}^{l}\right)^{2}\right]\end{aligned}$ \\
\hline$r=5$ & $\begin{aligned} F_{11}= & \left(v_{m}^{l+1}-v_{m+1}^{l}\right)\left[5-2\left(\left(v_{m}^{l+1}\right)^{2}+\left(v_{m+1}^{l}\right)^{2}\right)\left(5+12 v_{m}^{l+1} v_{m+1}^{l}\right.\right. \\
& \left.+5\left(v_{m}^{l+1}\right)^{2}\left(v_{m+1}^{l}\right)^{2}\right)+v_{m}^{l+1} v_{m+1}^{l}\left(40+76 v_{m}^{l+1} v_{m+1}^{l}+40\left(v_{m}^{l+1}\right)^{2}\left(v_{m+1}^{l}\right)^{2}\right. \\
& \left.\left.+5\left(v_{m}^{l+1}\right)^{3}\left(v_{m+1}^{l}\right)^{3}\right)+\left(v_{m}^{l+1}\right)^{4}+\left(v_{m+1}^{l}\right)^{4}\right] \\
F_{12}= & \left(1+v_{m}^{l+1} v_{m+1}^{l}\right)\left[1-10\left(\left(v_{m}^{l+1}\right)^{2}+\left(v_{m+1}^{l}\right)^{2}\right)\left(1+4 v_{m}^{l+1} v_{m+1}^{l}+\left(v_{m}^{l+1}\right)^{2}\left(v_{m+1}^{l}\right)^{2}\right)\right. \\
& +v_{m}^{l+1} v_{m+1}^{l}\left(24+76 v_{m}^{l+1} v_{m+1}^{l}+24\left(v_{m}^{l+1}\right)^{2}\left(v_{m+1}^{l}\right)^{2}+\left(v_{m}^{l+1}\right)^{3}\left(v_{m+1}^{l}\right)^{3}\right) \\
& \left.+5\left(v_{m}^{l+1}\right)^{4}+5\left(v_{m+1}^{l}\right)^{4}\right]\end{aligned}$ \\
\hline$r=6$ & $\begin{aligned} F_{11}= & 2\left(v_{m}^{l+1}-v_{m+1}^{l}\right)\left(1+v_{m}^{l+1} v_{m+1}^{l}\right)\left[3-\left(v_{m}^{l+1}\right)^{2}-\left(v_{m+1}^{l}\right)^{2}+8 v_{m}^{l+1} v_{m+1}^{l}\right. \\
& \left.+3\left(v_{m}^{l+1}\right)^{2}\left(v_{m+1}^{l}\right)^{2}\right]\left[1-3\left(v_{m}^{l+1}\right)^{2}-3\left(v_{m+1}^{l}\right)^{2}+8 v_{m}^{l+1} v_{m+1}^{l}+\left(v_{m}^{l+1}\right)^{2}\left(v_{m+1}^{l}\right)^{2}\right] \\
F_{12}= & \left(1+v_{m}^{l+1}-v_{m+1}^{l}+v_{m}^{l+1} v_{m+1}^{l}\right)\left(1-v_{m}^{l+1}+v_{m+1}^{l}+v_{m}^{l+1} v_{m+1}^{l}\right) \\
& {\left[1+4\left(v_{m}^{l+1}-v_{m+1}^{l}\right)+\left(v_{m}^{l+1}\right)^{2}+\left(v_{m+1}^{l}\right)^{2}+4 v_{m}^{l+1} v_{m+1}^{l}\left(v_{m}^{l+1}-v_{m+1}^{l}\right)+\left(v_{m}^{l+1}\right)^{2}\right.} \\
& \left.\left(v_{m+1}^{l}\right)^{2}\right]\left[1-4\left(v_{m}^{l+1}-v_{m+1}^{l}\right)+\left(v_{m}^{l+1}\right)^{2}+\left(v_{m+1}^{l}\right)^{2}-4 v_{m}^{l+1} v_{m+1}^{l}\left(v_{m}^{l+1}-v_{m+1}^{l}\right)\right. \\
& \left.+\left(v_{m}^{l+1}\right)^{2}\left(v_{m+1}^{l}\right)^{2}\right]\end{aligned}$ \\
\hline etc. & \\
\hline
\end{tabular}

Table 1: Explicit expressions of $F_{11}$ and $F_{12}$ in equation (2.18) with $r \in \mathbb{Z}^{+}$

\begin{tabular}{|c|c|}
\hline$r$ & Explicit expressions of $F_{11}$ and $F_{12}$ in equation (2.18) \\
\hline$r$-odd & $\begin{array}{l}F_{11}=\sum_{\substack{i=1 \\
i=\text { odd }}}^{|r|}(-1)^{\frac{i-1}{2}}\left(\begin{array}{c}|r| \\
i\end{array}\right)\left(\operatorname{sgn}(r)\left(v_{m}^{l+1}-v_{m+1}^{l}\right)\right)^{i}\left(1+v_{m}^{l+1} v_{m+1}^{l}\right)^{|r|-i} \\
F_{12}=\sum_{\substack{i=0 \\
i-\text { even }}}^{|r-1|}(-1)^{\left\lceil\frac{i-1}{2}\right\rceil}\left(\begin{array}{c}|r| \\
i\end{array}\right)\left(\operatorname{sgn}(r)\left(v_{m}^{l+1}-v_{m+1}^{l}\right)\right)^{i}\left(1+v_{m}^{l+1} v_{m+1}^{l}\right)^{|r|-i}\end{array}$ \\
\hline$r$-even & $\begin{array}{l}F_{11}=\sum_{\substack{i=1 \\
i=\text { odd }}}^{|r-1|}(-1)^{\frac{i-1}{2}}\left(\begin{array}{c}|r| \\
i\end{array}\right)\left(\operatorname{sgn}(r)\left(v_{m}^{l+1}-v_{m+1}^{l}\right)\right)^{i}\left(1+v_{m}^{l+1} v_{m+1}^{l}\right)^{|r|-i} \\
F_{12}=\sum_{\substack{i=0 \\
i-\text { even }}}^{|r|}(-1)^{\left\lceil\frac{i-1}{2}\right\rceil}\left(\begin{array}{c}|r| \\
i\end{array}\right)\left(\operatorname{sgn}(r)\left(v_{m}^{l+1}-v_{m+1}^{l}\right)\right)^{i}\left(1+v_{m}^{l+1} v_{m+1}^{l}\right)^{|r|-i}\end{array}$ \\
\hline
\end{tabular}

Table 2: Explicit expressions of $F_{11}$ and $F_{12}$ in equation (2.18) with $r \in \mathbb{Z} \backslash\{0\}$. 
where $\left\lceil\frac{i-1}{2}\right\rceil$ is the smallest integer greater than or equal to $\frac{i-1}{2}$ and

$$
\operatorname{sgn}(r)=\left\{\begin{array}{r}
1, \text { if } r>0 \\
0, \text { if } r=0 \\
-1, \text { if } r<0
\end{array}\right.
$$

It is appropriate to mention that Levi and Scimiterna [9] have derived a set of necessary conditions for a nonlinear $P \Delta \Delta E$ (2.4) to be linearizable [9] and we checked that equation (2.18) satisfies those conditions [9].

\section{Reductions to ordinary difference equations}

In this section we would like to show that how periodic reduction of the obtained nonlinear $P \Delta \Delta E$ 's result to higher order autonomous $O \Delta E$ 's. With this, we consider a solution $v_{m}^{l}$ of the nonlinear $P \Delta \Delta E$ (2.4) satisfying the periodicity property

$$
v_{m+z_{1}}^{l-z_{2}}=v_{l, m}=v_{n},
$$

where $\operatorname{gcd}\left(z_{1}, z_{2}\right)=1, z_{1}, z_{2} \in \mathbb{Z}$. Here $n=m z_{1}+l z_{2}$ and so

$$
v_{m+1}^{l}=v_{n+z_{1}}, \quad v_{m}^{l+1}=v_{n+z_{2}}, \quad v_{m+1}^{l+1}=v_{n+z_{1}+z_{2}}
$$

. As a consequence equation (2.4) becomes an $O \Delta E$ of order $\left(z_{1}+z_{2}\right)$, that is

$$
v_{n+z_{1}+z_{2}}=F\left(v_{n}, v_{n+z_{1}}, v_{n+z_{2}}\right) .
$$

We wish to report that the periodic reduction of each of the obtained $P \Delta \Delta E$ 's in section 2 namely (2.10), (2.12), (2.14) and (2.18) results to higher order $O \Delta E$ 's. We explain how to derive them below. To begin with we consider equation (2.10) which reduces into an $O \Delta E$ of order $\left(z_{1}+z_{2}\right)$

$$
v_{n+z+1}=\frac{h_{11} v_{n}^{3}+h_{12} v_{n}^{2}+h_{13} v_{n}+h_{14}}{h_{15} v_{n}^{3}+h_{16} v_{n}^{2}+h_{17} v_{n}+h_{18}},
$$

where $h_{1 i}^{\prime} s, i=1, \ldots 8$ are polynomials in $\left(v_{n+z_{2}}, v_{n+z_{1}}\right)$ given by,

$$
\begin{aligned}
h_{11}= & \alpha\left[1-\alpha^{6}+\alpha\left(\alpha^{4}-1\right) v_{n+z_{2}}+2 \alpha v_{n+z_{1}}-2 \alpha^{2}\right. \\
& \left.v_{n+z_{2}} v_{n+z_{1}}+\alpha^{2}\left(\alpha^{2}+1\right) v_{n+z_{1}}^{2}-2 \alpha^{3} v_{n+z_{2}} v_{n+z_{1}}^{2}\right] \\
h_{12}= & \alpha^{2}\left[\left(1-\alpha^{4}\right)+\alpha\left(\alpha^{2}-1\right) v_{n+z_{2}}+2 \alpha v_{n+z_{1}}-2 \alpha^{2} v_{n+z_{2}} v_{n+z_{1}}\right. \\
& \left.+2 \alpha^{2} v_{n+z_{1}}^{2}-\alpha\left(\alpha^{2}+1\right) v_{n+z_{2}} v_{n+z_{1}}^{2}\right] \\
h_{13}= & \alpha^{2}\left[\alpha\left(\alpha^{2}-1\right)-2 \alpha^{2} v_{n+z_{1}}+2 \alpha^{3} v_{n+z_{2}} v_{n+z_{1}}\right. \\
& \left.-\alpha\left(\alpha^{2}+1\right) v_{n+z_{1}}^{2}+\left(\alpha^{4}+1\right) v_{n+z_{2}} v_{n+z_{1}}^{2}\right] \\
h_{14}= & \alpha\left[\alpha^{2}\left(\alpha^{2}-1\right) v_{n+z_{2}}-2 \alpha^{4} v_{n+z_{1}}+2 \alpha^{5} v_{n+z_{2}} v_{n+z_{1}}\right. \\
& \left.-\alpha\left(\alpha^{4}+1\right) v_{n+z_{1}}^{2}+\left(\alpha^{6}+1\right) v_{n+z_{2}} v_{n+z_{1}}^{2}\right] \\
h_{15}= & \left(\alpha^{6}+1\right)-\alpha\left(\alpha^{4}+1\right) v_{n+z_{2}}+2 \alpha v_{n+z_{1}}-2 \alpha^{2} v_{n+z_{2}} v_{n+z_{1}} \\
& -\alpha^{2}\left(\alpha^{2}-1\right) v_{n+z_{1}}^{2}, \\
h_{16}= & \alpha\left[\left(\alpha^{4}+1\right)-\alpha\left(\alpha^{2}+1\right) v_{n+z_{2}}+2 \alpha v_{n+z_{1}}-2 \alpha^{2} v_{n+z_{2}} v_{n+z_{1}}\right. \\
& \left.-\alpha\left(\alpha^{2}-1\right) v_{n+z_{2}} v_{n+z_{1}}^{2}\right] \\
h_{17}= & \alpha\left[-\alpha\left(\alpha^{2}+1\right)+2 \alpha^{2}\left(v_{n+z_{2}}-v_{n+z_{1}}\right)+2 \alpha^{3} v_{n+z_{2}} v_{n+z_{1}}\right. \\
& \left.-\alpha\left(\alpha^{2}-1\right) v_{n+z_{1}}^{2}+\left(\alpha^{4}-1\right) v_{n+z_{2}} v_{n+z_{1}}^{2}\right] \\
h_{18}= & -2 \alpha^{3}+\alpha^{2}\left(\alpha^{2}+1\right) v_{n+z_{2}}-2 \alpha^{4} v_{n+z_{1}}+2 \alpha^{5} v_{n+z_{2}} v_{n+z_{1}} \\
& -\alpha\left(\alpha^{4}-1\right) v_{n+z_{1}}^{2}+\left(\alpha^{6}-1\right) v_{n+z_{2}} v_{n+z_{1}}^{2} .
\end{aligned}
$$


By assigning distinct values for $z_{1}$ and $z_{2}$ one can derive higher order $O \Delta E$ 's and its integrability is an open question. However equation (3.2) with $\alpha^{2}=-1$ or equation (2.12) becomes

$$
v_{n+z_{1}+z_{2}}=\frac{v_{n+z_{2}}-v_{n+z_{1}}+v_{n}\left(1+v_{n+z_{1}} v_{n+z_{2}}\right)}{\left(1+v_{n+z_{1}} v_{n+z_{2}}\right)-v_{n}\left(v_{n+z_{2}}-v_{n+z_{1}}\right)}
$$

which can be transformed into a linear $O \Delta E$

$$
\theta_{n+z_{1}+z_{2}}-\left(\theta_{n+z_{2}}-\theta_{n+z_{1}}\right)-\theta_{n}=p \pi, \theta_{n}=\tan ^{-1}\left(v_{n}\right), p \in \mathbb{Z}
$$

and hence the reduced $O \Delta E$ of order $\left(z_{1}+z_{2}\right)$ is linearizable and so integrable.

Similar conclusion can be arrived at for the reduced equation, obtained from equation (2.14), given by

$$
v_{n+z+1}=\frac{\tilde{h}_{11} v_{n}^{3}+\tilde{h}_{12} v_{n}^{2}+\tilde{h}_{13} v_{n}+\tilde{h}_{14}}{\tilde{h}_{15} v_{n}^{3}+\tilde{h}_{16} v_{n}^{2}+\tilde{h}_{17} v_{n}+\tilde{h}_{18}}
$$

where $\tilde{h}_{1 i}$ 's, $i=1, \ldots 8$ are polynomials in $\left(v_{n+z_{2}}, v_{n+z_{1}}\right)$ and its integrability is an open question. As before equation (3.6) with $\alpha^{2}=-1$ can be transformed into linear $O \Delta E$. Next, the $O \Delta E$ arising from equation (2.18) reads

$$
v_{n+z_{1}+z_{2}}=\frac{F_{11}\left(v_{n+z_{2}}, v_{n+z_{1}}\right)+v_{n} F_{12}\left(v_{n+z_{2}}, v_{n+z_{1}}\right)}{F_{12}\left(v_{n+z_{2}}, v_{n+z_{1}}\right)-v_{n} F_{11}\left(v_{n+z_{2}}, v_{n+z_{1}}\right)},
$$

where $F_{11}$ and $F_{12}$ are specific polynomials of degree $(2|r|-1)$ and $2|r|$ respectively and their explicit expression for different values of $r$ are given in Table 3 and Table 4.

Equation (3.7) can be transformed into a linear $O \Delta E$ of order $\left(z_{1}+z_{2}\right)$ with constant coefficients

$$
\theta_{n+z_{1}+z_{2}}-r\left(\theta_{n+z_{2}}-\theta_{n+z_{1}}\right)-\theta_{n}=p \pi, \theta_{n}=\tan ^{-1}\left(v_{n}\right), p \in \mathbb{Z}, r \in \mathbb{Z} \backslash\{0\}
$$

and so integrable.

We wish to add that the linearizable equation (3.7) also possesses more than one integrals for lower order with specific values of $r$. Some of them are as follows:

Case I: $r=1, z_{1}=1, z_{2}=2$

Equation (3.4) becomes a third order $O \Delta E$

$$
v_{n+3}=\frac{v_{n+2}-v_{n+1}+\left(1+v_{n+1} v_{n+2}\right) v_{n}}{\left(1+v_{n+1} v_{n+2}\right)-\left(v_{n+2}-v_{n+1}\right) v_{n}}
$$

which admits two integrals $J_{1}(n)$ and $J_{2}(n)$

$$
\begin{aligned}
& J_{1}(n)=\frac{1-v_{n} v_{n+2}}{1-v_{n} v_{n+2}+v_{n}+v_{n+2}}, \\
& J_{2}(n)=\frac{P_{1}(n)}{P_{2}(n)},
\end{aligned}
$$

where

$$
\begin{aligned}
P_{1}(n)= & \left(v_{n+1}^{2} v_{n+2}-v_{n+2}^{2}+v_{n+1} v_{n+2}+v_{n+1}\right) v_{n}^{2}+v_{n+1} v_{n+2}^{2}-v_{n+1}^{2} \\
& +\left(1+v_{n+1}\right) v_{n+2}+\left(1+v_{n+1}\right)\left(1+v_{n+2}\right)\left(1+v_{n+1} v_{n+2}\right) v_{n}, \\
P_{2}(n)= & {\left[v_{n+1} v_{n+2}\left(v_{n+1} v_{n+2}+v_{n+1}+1\right)+v_{n+1}^{2}+v_{n+1}+1\right] v_{n}^{2}+v_{n+1}^{2} v_{n+2}^{2}+1 } \\
& +\left(1+v_{n+1}\right)\left(1+v_{n+2}\right)\left(1+v_{n+1} v_{n+2}\right) v_{n}+\left(1+v_{n+1}\right)\left(v_{n+2}+v_{n+2}^{2}\right) .
\end{aligned}
$$




\begin{tabular}{|c|c|}
\hline$r$ & Explicit expressions of $F_{11}$ and $F_{12}$ in equation (3.7) \\
\hline$r=1$ & $\begin{array}{l}F_{11}=\left(v_{n+z_{2}}-v_{n+z_{1}}\right) \\
F_{12}=\left(1+v_{n+z_{2}} v_{n+z_{1}}\right)\end{array}$ \\
\hline$r=2$ & $\begin{array}{l}F_{11}=2\left(v_{n+z_{2}}-v_{n+z_{1}}\right)\left(1+v_{n+z_{2}} v_{n+z_{1}}\right) \\
F_{12}=\left(1+v_{n+z_{2}}-v_{n+z_{1}}+v_{n+z_{2}} v_{n+z_{1}}\right)\left(1-v_{n+z_{2}}+v_{n+z_{1}}+v_{n+z_{2}} v_{n+z_{1}}\right)\end{array}$ \\
\hline$r=3$ & $\begin{array}{l}F_{11}=\left(v_{n+z_{2}}-v_{n+z_{1}}\right)\left[3-\left(v_{n+z_{2}}\right)^{2}-\left(v_{n+z_{1}}\right)^{2}+8 v_{n+z_{2}} v_{n+z_{1}}+3\left(v_{n+z_{2}}\right)^{2}\left(v_{n+z_{1}}\right)^{2}\right] \\
F_{12}=\left(1+v_{n+z_{2}} v_{n+z_{1}}\right)\left[1-3\left(v_{n+z_{2}}\right)^{2}-3\left(v_{n+z_{1}}\right)^{2}+8 v_{n+z_{2}} v_{n+z_{1}}+\left(v_{n+z_{2}}\right)^{2}\left(v_{n+z_{1}}\right)^{2}\right]\end{array}$ \\
\hline$r=4$ & $\begin{aligned} F_{11}= & 2\left(v_{n+z_{2}}-v_{n+z_{1}}\right)\left(1+v_{n+z_{2}} v_{n+z_{1}}\right)\left(1+v_{n+z_{2}}-v_{n+z_{1}}+v_{n+z_{2}} v_{n+z_{1}}\right) \\
& \left(1-v_{n+z_{2}}+v_{n+z_{1}}+v_{n+z_{2}} v_{n+z_{1}}\right) \\
F_{12}= & {\left[1+2\left(v_{n+z_{2}}-v_{n+z_{1}}\right)-\left(v_{n+z_{2}}\right)^{2}-\left(v_{n+z_{1}}\right)^{2}+4 v_{n+z_{2}} v_{n+z_{1}}\right.} \\
& \left.+2 v_{n+z_{2}} v_{n+z_{1}}\left(v_{n+z_{2}}-v_{n+z_{1}}\right)+\left(v_{n+z_{2}}\right)^{2}\left(v_{n+z_{1}}\right)^{2}\right]\left[1-2\left(v_{n+z_{2}}-v_{n+z_{1}}\right)\right. \\
& -\left(v_{n+z_{2}}\right)^{2}-\left(v_{n+z_{1}}\right)^{2}+4 v_{n+z_{2}} v_{n+z_{1}}-2 v_{n+z_{2}} v_{n+z_{1}}\left(v_{n+z_{2}}-v_{n+z_{1}}\right) \\
& \left.+\left(v_{n+z_{2}}\right)^{2}\left(v_{n+z_{1}}\right)^{2}\right]\end{aligned}$ \\
\hline$r=5$ & $\begin{aligned} F_{11}= & \left(v_{n+z_{2}}-v_{n+z_{1}}\right)\left[5-2\left(\left(v_{n+z_{2}}\right)^{2}+\left(v_{n+z_{1}}\right)^{2}\right)\left(5+12 v_{n+z_{2}} v_{n+z_{1}}\right.\right. \\
& \left.+5\left(v_{n+z_{2}}\right)^{2}\left(v_{n+z_{1}}\right)^{2}\right)+v_{n+z_{2}} v_{n+z_{1}}\left(40+76 v_{n+z_{2}} v_{n+z_{1}}+40\left(v_{n+z_{2}}\right)^{2}\left(v_{n+z_{1}}\right)^{2}\right. \\
& \left.\left.+5\left(v_{n+z_{2}}\right)^{3}\left(v_{n+z_{1}}\right)^{3}\right)+\left(v_{n+z_{2}}\right)^{4}+\left(v_{n+z_{1}}\right)^{4}\right] \\
F_{12}= & \left(1+v_{n+z_{2}} v_{n+z_{1}}\right)\left[1-10\left(\left(v_{n+z_{2}}\right)^{2}+\left(v_{n+z_{1}}\right)^{2}\right)\left(1+4 v_{n+z_{2}} v_{n+z_{1}}\right.\right. \\
& \left.+\left(v_{n+z_{2}}\right)^{2}\left(v_{n+z_{1}}\right)^{2}\right)+v_{n+z_{2}} v_{n+z_{1}}\left(24+76 v_{n+z_{2}} v_{n+z_{1}}+24\left(v_{n+z_{2}}\right)^{2}\left(v_{n+z_{1}}\right)^{2}\right. \\
& \left.\left.+\left(v_{n+z_{2}}\right)^{3}\left(v_{n+z_{1}}\right)^{3}\right)+5\left(v_{n+z_{2}}\right)^{4}+5\left(v_{n+z_{1}}\right)^{4}\right]\end{aligned}$ \\
\hline$r=6$ & $\begin{aligned} F_{11}= & 2\left(v_{n+z_{2}}-v_{n+z_{1}}\right)\left(1+v_{n+z_{2}} v_{n+z_{1}}\right)\left[3-\left(v_{n+z_{2}}\right)^{2}-\left(v_{n+z_{1}}\right)^{2}+8 v_{n+z_{2}} v_{n+z_{1}}\right. \\
& \left.+3\left(v_{n+z_{2}}\right)^{2}\left(v_{n+z_{1}}\right)^{2}\right]\left[1-3\left(v_{n+z_{2}}\right)^{2}-3\left(v_{n+z_{1}}\right)^{2}+8 v_{n+z_{2}} v_{n+z_{1}}\right. \\
& \left.+\left(v_{n+z_{2}}\right)^{2}\left(v_{n+z_{1}}\right)^{2}\right] \\
F_{12}= & \left(1+v_{n+z_{2}}-v_{n+z_{1}}+v_{n+z_{2}} v_{n+z_{1}}\right)\left(1-v_{n+z_{2}}+v_{n+z_{1}}+v_{n+z_{2}} v_{n+z_{1}}\right) \\
& {\left[1+4\left(v_{n+z_{2}}-v_{n+z_{1}}\right)+\left(v_{n+z_{2}}\right)^{2}+\left(v_{n+z_{1}}\right)^{2}+4 v_{n+z_{2}} v_{n+z_{1}}\left(v_{n+z_{2}}-v_{n+z_{1}}\right)\right.} \\
& \left.+\left(v_{n+z_{2}}\right)^{2}\left(v_{n+z_{1}}\right)^{2}\right]\left[1-4\left(v_{n+z_{2}}-v_{n+z_{1}}\right)+\left(v_{n+z_{2}}\right)^{2}+\left(v_{n+z_{1}}\right)^{2}-4 v_{n+z_{2}} v_{n+z_{1}}\right. \\
& \left.\left(v_{n+z_{2}}-v_{n+z_{1}}\right)+\left(v_{n+z_{2}}\right)^{2}\left(v_{n+z_{1}}\right)^{2}\right]\end{aligned}$ \\
\hline etc., & \\
\hline
\end{tabular}

Table 3: Explicit expressions of $F_{11}$ and $F_{12}$ in equation (3.7) with $r \in \mathbb{Z}^{+}$

It is easy to check that equation (3.9) is measure preserving with measure $\frac{1}{P_{2}(n)}[31]$.

Case II: $r=1, z_{1}=1, z_{2}=3$

Here equation (3.4) becomes a fourth order $O \Delta E$

$$
v_{n+4}=\frac{v_{n+3}-v_{n+1}+\left(1+v_{n+1} v_{n+3}\right) v_{n}}{\left(1+v_{n+1} v_{n+3}\right)-\left(v_{n+3}-v_{n+1}\right) v_{n}}
$$

which admits two integrals $J_{1}(n)$ and $J_{2}(n)$

$$
\begin{aligned}
& J_{1}(n)=\frac{1-v_{n} v_{n+3}}{1-v_{n} v_{n+3}+v_{n}+v_{n+3}}, \\
& J_{2}(n)=\frac{Q_{1}(n)+Q_{2}(n)}{-Q_{1}(n)+Q_{2}(n)},
\end{aligned}
$$




\begin{tabular}{|c|c|}
\hline$r$ & Explicit expressions of $F_{11}$ and $F_{12}$ in equation (3.7) \\
\hline$r$-odd & $\begin{array}{l}F_{11}=\sum_{\substack{i=1 \\
i \text {-odd }}}^{|r|}(-1)^{\frac{i-1}{2}}\left(\begin{array}{c}|r| \\
i\end{array}\right)\left(\operatorname{sgn}(r)\left(v_{n+z_{2}}-v_{n+z_{1}}\right)\right)^{i}\left(1+v_{n+z_{2}} v_{n+z_{1}}\right)^{|r|-i} \\
F_{12}=\sum_{i=0}^{|r-1|}(-1)^{\left\lceil\frac{i-1}{2}\right\rceil}\left(\begin{array}{c}|r| \\
i\end{array}\right)\left(\operatorname{sgn}(r)\left(v_{n+z_{2}}-v_{n+z_{1}}\right)\right)^{i}\left(1+v_{n+z_{2}} v_{n+z_{1}}\right)^{|r|-i}\end{array}$ \\
\hline$r$-even & $\begin{array}{l}F_{11}=\sum_{\substack{i=1 \\
i-\text { odd }}}^{|r-1|}(-1)^{\frac{i-1}{2}}\left(\begin{array}{c}|r| \\
i\end{array}\right)\left(\operatorname{sgn}(r)\left(v_{n+z_{2}}-v_{n+z_{1}}\right)\right)^{i}\left(1+v_{n+z_{2}} v_{n+z_{1}}\right)^{|r|-i} \\
F_{12}=\sum_{i=0}^{|r|}(-1)^{\left\lceil\frac{i-1}{2}\right\rceil}\left(\begin{array}{c}|r| \\
i\end{array}\right)\left(\operatorname{sgn}(r)\left(v_{n+z_{2}}-v_{n+z_{1}}\right)\right)^{i}\left(1+v_{n+z_{2}} v_{n+z_{1}}\right)^{|r|-i}\end{array}$ \\
\hline
\end{tabular}

Table 4: Explicit expressions of $F_{11}$ and $F_{12}$ in equation (3.7) with $r \in \mathbb{Z} \backslash\{0\}$.

where

$Q_{1}(n)=\left(v_{n}+v_{n+3}\right)\left(v_{n+2} v_{n+3}+1\right)\left(v_{n+1} v_{n}+1\right)\left(v_{n+2} v_{n+1}+1\right)$, $Q_{2}(n)=\left(1+v_{n}^{2}\right)\left(1+v_{n+1}^{2}\right)\left(1+v_{n+2}^{2}\right)\left(1+v_{n+3}^{2}\right)$.

We have verified that equation (3.10) is a measure preserving one with measure $\frac{1}{\left[-Q_{1}(n)+Q_{2}(n)\right]}$ [32].

Case III: $r=2, z_{1}=1, z_{2}=2$

Equation (3.7) becomes a third order $O \Delta E$

$$
v_{n+3}=\frac{F_{11}\left(v_{n+2}, v_{n+1}\right)+v_{n} F_{12}\left(v_{n+2}, v_{n+1}\right)}{F_{12}\left(v_{n+2}, v_{n+1}\right)-v_{n} F_{11}\left(v_{n+2}, v_{n+1}\right)}
$$

where

$F_{11}=2\left(v_{n+2}-v_{n+1}\right)\left(1+v_{n+1} v_{n+2}\right)$,

$F_{12}=\left(1+v_{n+2}-v_{n+1}+v_{n+1} v_{n+2}\right)\left(1-v_{n+2}+v_{n+1}+v_{n+1} v_{n+2}\right)$.

which admits two integrals $J_{1}(n)$ and $J_{2}(n)$ given by

$$
\begin{aligned}
& J_{1}(n)=\frac{2\left[\left(v_{n+1}-1\right)\left(v_{n}+v_{n+2}\right)-\left(v_{n+1}+1\right)\left(v_{n} v_{n+2}-1\right)\right]}{v_{n+1}\left(v_{n}+v_{n+2}\right)-\left(v_{n} v_{n+2}-1\right)}, \\
& J_{2}(n)=\frac{R_{1}(n)}{R_{2}(n)}
\end{aligned}
$$

where

$$
\begin{aligned}
R_{1}(n)= & {\left[\gamma_{3}(n) v_{n+2}^{2}-\gamma_{2}(n) v_{n+2}+\gamma_{1}(n)\right] v_{n}^{2}+\left[-\gamma_{2}(n)\left(v_{n+2}^{2}-1\right)-4 v_{n+1} v_{n+2}\right] v_{n} } \\
& +\left[\gamma_{1}(n) v_{n+2}^{2}+\gamma_{2}(n) v_{n+2}+\gamma_{3}(n)\right], \\
R_{2}(n)= & -\left[\gamma_{1}(n) v_{n+2}^{2}+\gamma_{2}(n) v_{n+2}+\gamma_{3}(n)\right] v_{n}^{2}+\left[-\gamma_{2}(n)\left(v_{n+2}^{2}-1\right)-4 v_{n+1} v_{n+2}\right] v_{n} \\
& -\left[\gamma_{3}(n) v_{n+2}^{2}-\gamma_{2}(n) v_{n+2}+\gamma_{1}(n)\right], \\
\gamma_{1}(n)= & -\left(v_{n+1}^{2}+v_{n+1}+1\right), \gamma_{2}(n)=\left(v_{n+1}^{2}-1\right), \\
\gamma_{3}(n)= & \left(-v_{n+1}^{2}+v_{n+1}-1\right) .
\end{aligned}
$$

We have verified that equation (3.10) is measure preserving with measure $\frac{1}{R_{2}(n)}$ [31].

Similarly higher order $O \Delta E$ 's can be obtained by taking different values for $z_{1}$ and $z_{2}$ in equation (3.7) which may admit more than one integral. 


\section{Summary and Concluding Remarks}

In this article, a systematic investigation has been made to derive autonomous nonlinear $P \Delta \Delta E$ 's admitting specific Lax representation. In Section 2, we have derived two nonlinear $P \Delta \Delta E$ 's equations (2.10) and (2.14) possessing Lax pair but not belonging to QRT type. Also we have derived another nonlinear $P \Delta \Delta E$ (2.18) which is a QRT type and shown that it is linearizable with $r \in \mathbb{Z} \backslash\{0\}$. We would like to mention that nonlinear $P \Delta \Delta E$ 's (2.10) and (2.14) fall into equation (2.18) when $r=1$ and $r=2$ respectively, for a particular parametric restriction. Equation (2.12) gives a particular case of equation (2.18) when $r=1$.

In Section 3, we have shown how $O \Delta E$ 's of order $\left(z_{1}+z_{2}\right)$ namely equations (3.2), (3.6) and (3.7) can be derived from each of the identified nonlinear $P \Delta \Delta E$ 's (2.10), (2.14) and (2.18) respectively. Among them, the reduced equations (3.2) and (3.6) obtained from equations (2.10) and (2.14) respectively are nonintegrable in general and their integrability nature is under investigation. The reduced equation of order $\left(z_{1}+z_{2}\right)$ obtained from equation (2.18), given in equation (3.7) can be transformed into a linear $O \Delta E$ (3.8) ensuring its integrability. Further we have discussed the integrability of lower orders of equation (3.7) in three different cases giving different values for $z_{1}$ and $z_{2}$. We obtain one third order $O \Delta E$ each in Case I and III, from equation (3.7), namely equation (3.9) and equation (3.11) respectively, admitting two integrals. Next in Case II, from equation (3.7) we get a fourth order $O \Delta E$ (3.10) admitting two integrals.

Equations (3.9), (3.10) and (3.11) are measure preserving and the corresponding measures are given in each case. We would like to caution the reader that the analysis carried out in this article is not an exhaustive one. It is also interesting to find the most general form of Lax integrable and linearizable nonlinear $P \Delta \Delta E$ 's with the above mentioned global transformation which is under investigation. In addition examining the corresponding $O \Delta E$ 's, obtained through periodic reductions of the $P \Delta \Delta E$ 's, for integrability is also under investigation.

\section{Acknowledgement}

One of the authors(G.N) wishes to thank the Council of Scientific and Industrial Research (CSIR), Government of India, New Delhi for providing financial support in the form of Junior Research Fellowship.

\section{References}

[1] M. J. Ablowitz and J. F. Ladik, A nonlinear difference scheme and inverse scattering, Stud. Appl. Math. 55 (1976) 213-229.

[2] M. J. Ablowitz and J. F. Ladik, Nonlinear differential-difference equations and Fourier analysis, $J$. Math. Phys. 17 (1976) 1011-1018.

[3] M. J. Ablowitz and J. F. Ladik, On the solution of a class of nonlinear partial difference equations, Stud. Appl. Math. 57 (1977) 1-12.

[4] V. Adler, A. Bobenko and Yu. B. Suris, Classification of Integrable Equations on Quad-Graphs. The Consistency Approach Commun. Math. Phys. 233 (2003) 513-543.

[5] V. I. Arnold, Mathematical Methods of Classical Mechanics (Berlin: Springer, 1978).

[6] M. Bellon and C. M. Viallet, Algebraic entropy, Commun.Math. Phys. 204 (1999) 425-437.

[7] M. Brushi, O. Ragnisco, P. M. Santini and Gui Zhang Tu, Integrable symplectic maps Physica D 49 (1991) 273-294.

[8] H. W. Capel, F. W. Nijhoff and V. G. Papageorgiou, Complete integrability of Lagrangian mappings and lattices of KdV type, Phys. Lett. A 155 (1991) 377. 
[9] D. Levi and C. Scimiterna, Linearizability of Nonlinear Equations on a Quad-Graph by a point, Two points and Generalized Hopf-Cole Transformations, SIGMA 7 (2011) 079.

[10] B. Grammaticos, A. Ramani and V. Papageorgiou, Do integrable mappings have the Painlevé property? Phys. Rev. Lett. 67 (1991) 1825-1828.

[11] B. Grammaticos, Y. Kosmann-Schwarzbach and T. Tamizhmani, Discrete Integrable Systems (Berlin: Springer, 2004).

[12] B. Grammaticos, A. Ramani, Integrable mappings with transcendental invariants Commun. Nonlinear Sci. Numer. Simul. 12 (2007) 350-356.

[13] R. Hirota, Nonlinear partial difference equations I-III J. Phys. Soc. Jpn. 43 (1977) 1424-1433, 20742089.

[14] R. Hirota, K. Kimura and H. Yahagi, How to find the conserved quantities of nonlinear discrete equations J. Phys. A: Math. Gen. 34 (2001) 10377-10386.

[15] J. Hietarinta and C. Viallet, Singularity confinement and chaos in discrete systems Phys. Rev. Lett. 81 (1998) 325-328.

[16] J. Hietarinta, A new two-dimensional lattice model that is 'consistent around a cube' J. Phys. A: Math. Gen. 37 (2004) L67.

[17] P. E. Hydon, Conservation laws of partial difference equations with two independent variables J. Phys. A: Math. Gen. 34 (2001) 10347-10355.

[18] N. Joshi, D. Burtonclay and R. G. Halburd, Nonlinear nonautonomous discrete dynamical system from a general discrete isomonodromy problem Lett. Math. Phys. 26 (1992) 123.

[19] K. Maruno, K. Kajiwara, S. Nakao and M. Oikawa, Bilinearization of Discrete Soliton Equations and Singularity Confinement arXiv:sol-int/9610005v1 (1996).

[20] E. C. McMillan, Topics in Physics, Brittin W E and Odabasi H (Boulder: Colorado University Press, 1971) p.219.

[21] V. G. Papageorgiou, F. W. Nijhoff and H. W. Capel, Integrable mappings and nonlinear integrable lattice equations Phys. Lett. A 147 (1990) 106-114.

[22] H. Peter, O. Rojas and G. R. W. Quispel, Closed-form expressions for integrals of MKdV and SineGordon maps J. Phys. A: Math. Theor. 40 (2007) 12789-12798.

[23] G. R. W. Quispel, J. A. G. Roberts and C. J. Thompson, Integrable mappings and soliton equations I, Phys. Lett. A 126 (1988) 419-421.

[24] G. R. W. Quispel, J. A. G. Roberts and C. J. Thompson, Integrable mappings and soliton equations II, Physica D 34 (1989) 183-192

[25] G. R. W. Quispel, H. W. Capel, V. G. Papageorgiou and F. W. Nijhoff, Integrable mappings derived from soliton equations Physica A 173 (1991) 243-266.

[26] G. R. W. Quispel, H. W. Capel and J. A. G. Roberts, Duality of discrete integrable systems J. Phys. A: Math. and Gen. 38 (2005) 3965-3980.

[27] A. Ramani, B. Grammaticos and S. Lafortune, The discrete Chazy III system of Labtunie Conte is not integrable J. Phys. A: Math. and Gen. 35 (2002) 7943-7946.

[28] J. A. G. Roberts and G. R. W. Quispel, Creating and relating 3-dimensional integrable maps J. Phys. A: Math. and Gen. 39 (2006) L605-L615.

[29] R. Sahadevan and S. Balakrishnan, Complete integrability of two coupled discrete modified Kortewegde Vries equations J. Phys. A: Math. Theor. 42 (2009) 415208 (11 pages).

[30] R. Sahadevan and G. Nagavigneshwari, New Lax integrable nonlinear partial difference equations, submitted to J. Math. Phys.

[31] R. Sahadevan and C. Uma Maheswari, Third order difference equations with two rational integrals, $J$. Phys. A: Math. and Theor. 42 (2009) 454017(11pp).

[32] C. Uma Maheswari and R. Sahadevan, Integrable fourth-order difference equations, J. Phys. A: Math. and Theor. 43 (2010) 235203(13pp).

[33] Tsuda, Integrable mappings via rational elliptic surfaces J. Phys. A: Math. and Gen. 37 (2004) 27212730.

[34] A. P. Veselov, Integrable maps Russian Math. Surveys 46 (1991) 1-51. 